\title{
Multidrug resistant Gram-negative infections in neonatology
}

\author{
Griselda Berberian, M.D. ${ }^{a}$, Martín Brizuela, M.D. ${ }^{a}$, María T. Rosanova, M.D. ${ }^{a}$ \\ Mónica Travaglianti, Biochemist ${ }^{b}$, Alejandra Mastroiani, Lab Technician ${ }^{c}$, \\ Vanesa Reijtman, M.D. ', Graciela Fiorili, M.D. ${ }^{c}$, Dora Santa Cruz, B.S. ${ }^{d}$ and \\ Graciela Castro, M.D. ${ }^{e}$
}

\begin{abstract}
Introduction. Multidrug resistant Gramnegative (MDRGN) infections are an increasing problem in neonatal intensive care units. The objective of this study was to establish the epidemiological, clinical, microbiological, and evolutionary characteristics of carbapenemresistant MDRGN infections and the risk factors for them at the Division of Neonatology of a tertiary care hospital.

Population and method. A retrospective cohort study was done in this Division in patients with a documented MDRGN infection between 4/24/2013 and 4/29/2015.

Results. Twenty-one patients were included. Their median gestational age and birth weight were 35 weeks and $2070 \mathrm{~g}$, respectively. Eighteen patients $(86 \%)$ had a positive blood culture; the most commonly isolated microorganism was Acinetobacter baumannii (17 patients, $81 \%$ ), followed by carbapenemase-producing Klebsiella pneumoniae (3 patients, $14 \%$ ) and Enterobacter cloacae (1 patient, $5 \%$ ).

The median age at diagnosis was 28 days and all patients had risk factors for infection, including surgery, assisted mechanical ventilation, parenteral nutrition, central venous line, and antibiotics. The definite antibiotic therapy included colistin in all cases; in combination, in $84 \%$. Five patients (24\%) died due to theinfection. Prematurity and a birth weight $<2000 \mathrm{~g}$ were statistically significant risk factors associated with mortality ( $p=0.03$ and 0.01 , respectively). Conclusion. MDRGNinfections wereobserved in patients with predisposing factors. Acinetobacter baumannii was the main etiologic agent. Mortality was high and related to prematurity and a low birth weight.

Key words: Multidrug bacterial resistance, Gramnegative bacteria, newborn.
\end{abstract}

http: / / dx.doi.org/10.5546/ aap.2019.eng.6

E-mail address:

Griselda Berberian, M.D.: griselberberian@yahoo. com.ar

Funding:

None.

Conflict of interest:

None.

Received: 1-11-2018

Accepted: 7-12-2018

\section{INTRODUCTION}

Due to the greater survival and complexity in newborn care, multidrug resistant microorganisms are an emerging problem in neonatal care units. Increasing antibiotic resistance, together with restrictions in new drug development, especially in the neonatal population, makes multidrug resistant bacterial infections an ongoing problem that increases the risk for morbidity and mortality. ${ }^{1}$ An adequate empirical therapy for severe bacterial infections is critically relevant to reduce mortality, and carbapenems are still the initial treatment of choice for hospitalacquired infections in most neonatal intensive care units (NICUs).

The objective of this study was to establish the epidemiological, clinical, and microbiological characteristics of patients with carbapenem-resistant MDRGN infection, their risk factors for infection, and mortality at the NICU of a tertiary care hospital, which would allow us to recognize, manage, and prevent these infections rapidly.

\section{POPULATION AND METHODS}

This was a descriptive, retrospective, and observational study. Patients hospitalized at the NICU of Hospital de Pediatría "Profesor Dr. Juan P. Garrahan" with To cite: Berberian G, Brizuela M, Rosanova MT, a carbapenem-resistant MDRGN Travaglianti M, et al. Multidrug resistant Gram-
negative infections in neonatology. Arch Argent Pediatr negative infections in neonatology. Arch Argent Pediatr
2019;117(1):6-11. $4 / 25 / 2013$ (date when the microorganism was first isolated at the NICU) and 4/29/2015 were included.

The study site was a tertiary care children's hospital located in Buenos Aires, Argentina. This hospital does 
not have a maternity ward; the neonatal care unit has 60 beds for patients who are referred, in most cases, for surgery.

Patients with isolation of MDRGN bacilli with intrinsic resistance to carbapenems were excluded. Intrinsic resistance was defined as that naturally occurring in the microorganism.

MDRGN bacilli were defined based on the consensus by the Centers for Diseases Control and Prevention (CDC) and the European Center for Diseases Prevention and Control (ECDC) as microorganisms resistant to one or more drugs in three or more antibiotic groups, including those resistant to carbapenems. ${ }^{2}$

Microbiology: Sensitivity tests were done using the disc diffusion and Etest in MüellerHinton agar method and the Vitek 2 Compact automated method (Biomerieux) as per the recommendations of the Clinical and Laboratory Standards Institute (CLSI). ${ }^{3}$

The following outcome measures were assessed: birth weight, gestational age (GA), sex, underlying disease, risk factors for infection such as surgery, assisted mechanical ventilation (AMV), urinary catheter, parenteral nutrition, vascular lines, prior antibiotic use, age at the time of infection, clinical characteristics, microbiological laboratory analysis, treatment, course, and the presence of enteral colonization with the same microorganism.

Infection-associated mortality was defined as that occurred within one month after starting an antibiotic therapy adequate for the isolated microorganism as per the antibiogram sensitivity results.

Statistical analysis: Data were processed using the Epi-Info 6.0 software. Continuous outcome measures were reported as mean or median and range. Categorical outcome measures were expressed in number and percentage, and a $p$ value $\leq 0.05$ was considered statistically significant. A univariate analysis was done to assess the risk factors for infection and mortality.

\section{ETHICAL ASPECTS}

The study was approved for publication by the Hospital Ethics Committee of Hospital de Pediatría "J. P. Garrahan".

\section{RESULTS}

A total of 21 patients with carbapenemresistant MDRGN infection were included; 20 of which had acquired it at the NICU of Hospital de Pediatría “J. P. Garrahan".
Patients' median GA was 35 weeks (range: 28-39 weeks) and their median birth weight was 2070 g (range: $920-3850$ g); 14 patients $(66 \%)$ were males. Twelve patients $(57 \%)$ were preterm infants (GA < 37 weeks), and 6/12 (50\%) had a birth weight of less than $1500 \mathrm{~g}$.

All patients had an underlying disease. Most of them, 14 patients $(67 \%)$, had gastrointestinal tract involvement (gastroschisis, esophageal atresia, anorectal malformation, intestinal atresia, necrotizing enterocolitis); $12 / 14$ (86\%) had congenital malformations that required surgery.

Every patient had risk factors for infection. The presence of a central venous line (CVL) and prior antibiotic use were observed in the 21 patients $(100 \%)$. The median length of CVL use was 29 days (range: 10-114 days). A history of surgery and AMV and parenteral nutrition requirements were observed in $18(86 \%)$ and $20(95 \%)$ patients, respectively (Table 1).

A history of prior infection was recorded in 18 patients ( $85 \%)$, and it was microbiologically documented in 12 of them $(57 \%)$. The median age at diagnosis was 28 days (range: 8-90 days). The diagnosis was primary bacteremia in 12 patients $(57 \%)$, bacteremia secondary to enteritis ( 5 patients, $24 \%$ ), urinary tract infection (3 patients, $14 \%$ ), endocarditis (1 patient, $5 \%$ ).

TABLE 1: Population and risk factors for infection

\begin{tabular}{|c|c|}
\hline \multicolumn{2}{|l|}{ Outcome measures } \\
\hline Birth weight (median) & $\begin{array}{l}2070 \text { grams } \\
\text { (r: 920-3850) }\end{array}$ \\
\hline Gestational age (median) & $\begin{array}{l}35 \text { weeks } \\
\text { (r: 28-39) }\end{array}$ \\
\hline Male sex, $\mathrm{N}(\%)$ & $14(66)$ \\
\hline Prematurity (GA < 37 weeks), $\mathrm{N}(\%)$ & $12(57)$ \\
\hline Underlying disease, $\mathrm{N}(\%)$ & $21(100)$ \\
\hline Age at diagnosis (median), days & $28(8-90)$ \\
\hline \multicolumn{2}{|l|}{ Risk factors for infection, $\mathrm{N}(\%)$} \\
\hline Central venous line & $21(100)$ \\
\hline Prior antibiotic use & $21(100)$ \\
\hline Underlying disease & $21(100)$ \\
\hline Assisted mechanical ventilation & $20(95)$ \\
\hline Parenteral nutrition & $20(95)$ \\
\hline Prior infection & $18(86)$ \\
\hline Prior surgery & $18(86)$ \\
\hline Gastrointestinal disease & $14(67)$ \\
\hline
\end{tabular}

GA: gestational age. 
Microorganisms were isolated in the blood culture of 18 patients $(86 \%)$, in the urine culture of $2(9.5 \%)$, and in the peritoneal fluid culture of $1(4.5 \%)$. In 3 patients (14\%), MDRGN bacilli were isolated together with other microorganisms, forming a polymicrobial flora.

The main isolated microorganisms were Acinetobacter baumannii in 17 patients $(81 \%)$, carbapenemase-producing Klebsiella pneumoniae (CPKP) in $3(14 \%)$, and Enterobacter cloacae in $1(5 \%)$. All children in this series underwent an intestinal colonization test for MDRGN; results showed that 7 (33\%) also presented intestinal colonization with the same microorganism: 5 with Acinetobacter baumannii and 2 with CPKP (Table 2).

Thirteen patients $(63 \%)$ had received prior carbapenem treatment (meropenem) for a mean of 14 days (range: 4-46 days).

The targeted antibiotic therapy based on microbiological sensitivity consisted in colistin in 19 patients (90\%); in 16/19 patients $(84 \%)$ it was combined with ampicillin-sulbactam for Acinetobacter baumannii and with meropenem for $\mathrm{CPKP}$ as per the synergy testing results. Colistin was not administered to 2 patients $(10 \%)$ because they died before microbiological isolation was confirmed and an adequate antibiotic therapy was indicated. The 3 patients who received colistin monotherapy had an adequate clinical course; Acinetobacter spp. had been isolated in them. The median antibiotic therapy duration was 14 days (range: 1-48 days). The median length of stay was 86 days (range: $10-455$ days).

Five patients $(24 \%)$ died due to the MDRGN infection (mortality: $24 \%$ ); 2 of them did not live to receive the adequate therapy. The other 3 patients were on day 1,16 , and 21 of colistinmeropenem combination therapy, respectively.

TABLE 2. Microbiological findings

\begin{tabular}{lc}
\hline & $\mathbf{N}(\%)$ \\
\hline Isolation site & $18(86)$ \\
Blood culture & $2(9,5)$ \\
Urine culture & $1(4,5)$ \\
Peritoneal fluid culture & \\
Isolated microorganisms & $17(81)$ \\
Acinetobacter baumannii & $3(14)$ \\
CPKP & $1(5)$ \\
Enterobacter cloacae & $7(33,3)$ \\
Prior gastrointestinal colonization &
\end{tabular}

CPKP: Carbapenemase-producing Klebsiella pneumoniae.
Four of the deceased patients $(80 \%)$ were males and had gastrointestinal disease. Acinetobacter baumannii was isolated in 3 patients, and CPKP, in the other 2. All deceased patients were preterm infants with a median GA of 31 weeks and a birth weight of $1521 \mathrm{~g}$.

The univariate analysis showed that prematurity and a birth weight $<2000 \mathrm{~g}$ were risk factors associated with mortality $(p=0.03$ and $0.01)$, respectively.

\section{DISCUSSION}

Infections are one of the most common complications in the NICU; they lead to an increase in morbidity and mortality, an extended length of stay, and higher hospital costs, especially in the case of multidrug resistant microorganisms. ${ }^{1}$

Although, in general, the most common microorganisms observed in the NICU are Gram-positive cocci, the incidence of Gramnegative infections and multidrug resistant microorganisms has increased in recent years. ${ }^{4}$ The emergence of MDRGN bacilli, especially those resistant to carbapenems, is a diagnostic and therapeutic challenge in the neonatal population because the evidence is limited to case series or small outbreaks, in addition to the scarce availability of treatments with proven effectiveness and safety, as well as the multiple hurdles caused by the implementation of adequate isolation measures at the NICU. Most recommendations have been extrapolated from the experience in adults. 5,6

MDRGN infections have been mainly reported in preterm newborn infants and in patients with risk factors, such as the presence of an underlying disease, extended length of stay, surgery, prior antibiotic use, and invasive procedures, like CVL, $\mathrm{AMV}$, and urinary catheter. ${ }^{7,8}$

All patients in this series had one or more risk factors for MDRGN infection, and more than half were preterm infants. In maternity centers where preterm infants are monitored, bronchopulmonary dysplasia and perinatal neurological disease were the most commonly associated conditions. ${ }^{9}$

Prior antibiotic use, especially third-generation cephalosporins and carbapenems, is key for the development of resistance. . $^{10,11}$

The use of broad-spectrum antibiotics and carbapenems has increased in recent years due to the growth of extended spectrum betalactamase (ESBL)-producing bacilli, which has 
favored the development of carbapenem-resistant MDRGN bacilli. ${ }^{12}$ These findings are consistent with those of our study, where all children were observed to have a history of prior antibiotic use. Meropenem was the most commonly used antibiotic based on the epidemiology observed at the NICU and was part of the initial empirical therapy for suspected nosocomial sepsis.

The development of MDRGN at the NICU was directly related to a greater antibiotic use due to the higher number of hospital-acquired Gramnegative infections during such period. The most frequent presentations of MDRGN infections were sepsis and bacteremia (68\%), followed by urinary tract infection and pneumonia, among others. ${ }^{13}$ In this series, primary bacteremia was the most common clinical presentation, followed by enteritis and urinary tract infection.

The epidemiology of carbapenem-resistant MDRGN bacilli varies depending on the series. Acinetobacter baumannii and Klebsiella pneumoniae were the most frequently reported microorganisms. ${ }^{14}$ In our series, Acinetobacter baumannii was the most common one, followed by CPKP and Enterobacter cloacae, in agreement with the bibliography.

The antibiotic therapy of choice and the need for a combination with other antimicrobials in MDRGN infections have been the subject of controversy. Carbapenem use has increased in the past 10 years due to the dissemination of ESBL+ Gram-negative bacilli; this has favored the emergence of carbapenemase-producing genes (KPC, NDM, OXA-48, among others), which facilitate inter-human and inter-species dissemination of resistant bacteria. ${ }^{15}$ Carbapenemresistant MDRGN infections are resistant to all beta-lactams, including carbapenems and their combination with beta-lactamase inhibitors (except for ceftazidime-avibactam) and usually coexist with resistance to other antibiotics, such as aminoglycosides and fluoroquinolones, which restricts treatment options. The resistance mechanism may be the result of the production of carbapenemases or other mechanisms, such as a reduced permeability or drug efflux mechanisms. ${ }^{16}$

The emergence and dissemination of carbapenem-resistant MDRGN bacilli at the NICUs have resulted in the need to use old antibiotics, such as polymyxins (colistin/ polymyxin B) and fosfomycin, or others like tigecycline, to which they are usually sensitive. ${ }^{11}$

Colistin is a drug that has resurfaced for parenteral use in the past decade in relation to the emergence of carbapenem-resistant MDRGN infections and that, many times, has become the only therapeutic option. There is scarce experience with its use in newborn infants but, with controlled doses and durations, it may be a safe and effective drug. Colistin in carbapenem-resistant MDRGN infections may or not be combined with another agent, such as carbapenem, ampicillin-sulbactam/ aminoglycosides, depending on each case. ${ }^{17,18}$

The experience with the use of fosfomycin in newborn infants is scarce and should be considered as a last resource for children for whom there are no other therapeutic options. The advantage of this broad-spectrum antibiotic is that it has adequate urine, plasma, lung, and cerebrospinal fluid levels and its low toxicity. ${ }^{19}$ Resistance has been described in relation to the use of fosfomycin as monotherapy so it should always be used in combination. ${ }^{20}$ Other drugs like tigecycline are used in older patients, but they are not recommended in the neonatal population due to their potential action on the growth plate..$^{16,19,20}$

New antibiotics are currently being developed for the treatment of these infections but only a few are targeted at carbapenem-resistant MDRGN bacilli, such as aztreonam-avibactam, ceftazidimeavivactam, meropenem + vaborbactam, and plazomicin. Only meropenem-vaborbactam is being developed with a safety and pharmacokinetic assessment for pediatric use, including newborn infants. ${ }^{21-23}$

The definite treatment is indicated based on microbiological sensitivity results, and synergy or additive testing for each specific situation. The definite antibiotic therapy in this series included colistin in all patients, except for 2 who died before the microbiological result was obtained and an adequate antibiotic could be indicated.

The combination with beta-lactamase inhibitors to strengthen colistin action in the case of Acinetobacter baumannii, which was the most commonly isolated microorganism, has been described as a synergistic combination in several clinical reports. ${ }^{24,25}$ The antibiotic combination of choice and its effect on the reduction of mortality in MDRGN infections is a controversial topic.

In a multicenter study in 480 patients published in 2017, the antibiotic combination was observed to have a protective effect on mortality, especially in patients with a severe condition at admission, while monotherapy was reserved for those with a low risk for mortality. ${ }^{26}$ 
Intestinal colonization with carbapenemresistant MDRGN bacilli is directly related to the length of stay and the exposure to carbapenem at the NICU, although there are no conclusive data about the clinical implications of such finding as a predictor of infection. ${ }^{27}$

An epidemiological surveillance study that looked for rectal colonization with resistant microorganisms showed an association between resistant bacteria colonization and the use of meropenem for more than 10 days in patients hospitalized at the NICU for more than 2 weeks. Among colonized patients, $47 \%$ developed an infection. ${ }^{28}$ Considering the heterogeneity and scarcity of available studies in newborn infants, it is not possible to make conclusive recommendations in colonized patients.

MDRGN infections are associated with a high risk for complications and death. In adult patients, mortality has been reported to be $40-65 \%$. A recent publication described the first estimation of neonatal deaths attributed to resistant infections, which are responsible for approximately $30 \%$ of all neonatal deaths caused by sepsis. ${ }^{29}$

In our series, mortality was $24 \%$ and related factors were prematurity and a birth weight $<2000 \mathrm{~g}(p=0.03$ and $p=0.01$, respectively).

Adequate prevention policies targeted at hospital-acquired infections, as well as special prevention measures against the transmission of multidrug resistant microorganisms, together with a rational antibiotic use, are key tools to prevent the dissemination of these infections. ${ }^{30}$

\section{CONCLUSIONS}

Carbapenem-resistant MDRGN infections were observed in patients with predisposing factors, such as underlying disease, CVL, and prior antibiotic use. Acinetobacter baumannii was the main etiologic agent. Mortality was high and related to prematurity and a low birth weight.

\section{REFERENCES}

1. Folgori L, Bielicki J, Heath P, Sharland M. Antimicrobialresistant Gram-negative infections in neonates: burden of disease and challenges in treatment. Curr Opin Infect Dis. 2017; 30(3):281-8.

2. Magiorakos A, Srinivasan A, Carey R, et al. Multidrugresistant, extensively drug-resistant and pandrug-resistant bacteria: an international expert proposal for interim standard definitions for acquired resistance. Clin Microbiol Infect. 2012; 18(3):268-81.

3. Protocolo de trabajo red WHONET Argentina. XVII taller WHONET-Argentina. Rosario, octubre de 2016. [Accessed on: July $\left.12^{\text {th }}, 2018\right]$. Available at: http:/ / antimicrobianos. com.ar/ATB / wp-content/ uploads / 2014/10/ ProtocoloWHONET-consensuado-2017-final.pdf.
4. Cohen-Wolkowiez M, Moran C, Benjamin DK, et al. Early and late onset sepsis in late preterm infants. Pediatr Infect Dis J. 2009; 28(12):1052-6.

5. Le Doare K, Bielicki J, Heath PT, Sharland M. Systematic review of antibiotic resistance rates among gram negative bacteria in children with sepsis in resource-limited countries. J Pediatric Infect Dis Soc. 2015; 4(1):11-20.

6. Petrosillo N, Giannella M, Lewis R, Viale P. Treatment of carbapenem-resistant klebsiella pneumoniae: the state of the art. Expert Rev Anti Infect Ther. 2013; 11(2):159-77.

7. Tsai MH, Chu SM, Hsu JF, et al. Risk factors and outcomes for multidrug resistant Gram-negative bacteremia in the NICU. Pediatrics. 2014; 133(2):e322-9.

8. Giuffre M, Geraci D, Bonura C, et al. The increasing challenge of multidrug-resistant Gram-negative bacilli: results of a 5-year active surveillance program in a neonatal intensive care unit. Medicine (Baltimore). 2016; 95(10):e3016.

9. Tsai MH, ChuSM, Hsu JF, et al. Polymicrobial bloodstream infection in neonates: microbiology, clinical characteristics and risk factors. PloS One. 2014; 9(1):e83082.

10. Levy Hara G, Gould I, Endimiani A, et al. Detection, treatment, and prevention of carbapenemase-producing Enterobacteriaceae: recommendations from an International Working Group. J Chemother. 2013; 25(3):129-40.

11. Gray W, Patel M. Management of antibiotic-resistant infection in the newborn. Arch Dis Child Educ Pract Ed. 2011; 96(4):122-7.

12. Chiotos K, Han JH, Tamma PD. Carbapenem-resistant Enterobacteriaceae infections in children. Curr Infect Dis Rep. 2016; 18(1):2.

13. UrzedoJE, Levenhagen MM, Pedroso RS, et al. Nosocomial infections in a neonatal intensive care unit during 16 years: 1997-2012. Rev Soc Bras Med Trop. 2014; 47(3):321-6.

14. Viswanathan R, Singh AK, Basu S, etal.Multi-drug resistant, non-fermenting, gram negative bacilli in neonatal sepsis in Kolkata, India: a 4-year study. Pediatr Int Child Health. 2014; 34(1):55-9.

15. Tzouvelekis LS, Markogiannakis A, Piperaki E, et al. Treating infections caused by carbapenemase-producing Enterobacteriaceae. Clin Microbiol Infect. 2014; 20(9):862-72.

16. Gray J, Ubhi H, Milner P. Antimicrobial treatment of serious gram negative infections in newborns. Curr Infect Dis Rep. 2014; 16(2):400.

17. Jajoo M, Kumar V, Jain M, et al. Intravenous Colistin Administration in neonates. Pediatr inf Dis J. 2011; 30(3): 218-21.

18. Ni W, Cai X, Wei C, et al. Efficacy of polymyxins in the treatment of carbapenem-resistant Enterobacteriaceae infections: a systematic review and meta-analysis. Braz J Infect Dis. 2015; 19(2):170-80.

19. Hsu AJ, Tamma PD. Treatment of multidrug-resistant Gram-negative infections in children. Clin Infect Dis. 2014; 58(10):1439-48

20. Tzialla C, Borghesi a, Serra G, et al. Antimicrobial therapy in neonatal intensive care unit. Ital J Pediatr. 2015; 41:27.

21. Antibiotics Currently in Clinical Development 2016. [Accessed on: July 12 ${ }^{\text {th }}, 2018$ ]. Available at: http:/ / www. pewtrusts.org/ / media / assets / 2016/12/antibiotics_ datatable_201612.pdf.

22. Iovleva A, Doi Y. Carbapenem-Resistant Enterobacteriaceae. Clin Lab Med. 2017; 37(2):303-15.

23. Dose-finding, pharmacokinetics, safety and tolerability of VABOMERE (meropenem-vaborbactam) in pediatric subjects with serious bacterial infections (TANGOKIDS). [Accessed on: July 12 ${ }^{\text {th }}, 2018$ ]. Available at: https:// clinicaltrials.gov/ct2/ show / NCT02687906.

24. Sakoulas G, Rose W, Berti A, et al. Classical $\beta$ lactamase inhibitors potentiate the activity of daptomycin against 
Methicilin-Resistant Staphylococcus aureus and colistin against Acinetobacter baumannii. Antimicrob Agents Chemother. 2017; 61(2):e01745-16.

25. Adnan S. Paterson D, Lipman J, Roberts JA. Ampicillin/ sulbactam: its potential use in treating infections in critically ill patients. Int J Antimicrob Agents. 2013; 42(5):384-9.

26. Gutiérrez-Gutiérrez B, Salamanca E, de Cueto $M$, et al. Effect of appropriate combination therapy on mortality of patients with bloodstream infections due to carbapenemaseproducing Enterobacteriaceae (INCREMENT): a retrospective cohort study. Lancet Infect Dis. 2017; 17(7):726-34.

27. Clock SA, Ferng YH, Tabibi S, et al. Colonization with antimicrobial-resistant Gram-negative bacilli at neonatal intensive care unit discharge. J Pediatr Infect Dis Soc. 2017; 6(3)219-26.
28. Debby BF, Ganor O, Yasmin M, et al. Epidemiology of carbapenem resistant Klebsiella pneumoniae colonization in an intensive care unit. Eur J Clin Microbiol Infect Dis. 2012; 31(8).1811-7.

29. Laxminarayan $R$, Matsoso $P$, Pant $S$, et al. Access to effective antimicrobials: a worldwide challenge. Lancet. 2016; 387(10014):168-75.

30. National Center for Emerging and Zoonotic Infectious Diseases. Facility Guidance for control of Carbapenemresistant Enterobacteriaceae. CRE Toolkit. Atlanta: CDC; 2015. [Accessed on: July 12 ${ }^{\text {th }}, 2018$ ]. Available at: www. cdc.gov/ hai/pdfs/ cre/CRE-guidance-508.pdf. 